\title{
Role of Growth Factors-rich Plasma (Activated Platelet- rich Plasma) in Androgenic Alopecia: Literature Review and Suggested Treatment Protocol
}

\author{
Sheher Bano and Tariq Mehmood Dar
}

\section{ABSTRACT}

Androgenetic alopecia (AGA) has become a prevalent hair fall dysfunction due to genetic as well as hormonal effects distinguished by androgenrelated gradual weakening of the scalp hair with a sequence. Around turn 60, 45 percent of males and 35 percent of females come down with AGA. Owing to the small number of successful AGA therapies, Platelet-rich Plasma (PRP) has been an attractive alternative therapeutic approach. Platelet-rich plasma (PRP) is being proposed for the incorporation of greater levels of growth factors and certain other biologically active substances in wounded lesions to achieve the specific healing objectives. PRP is already being included in dentistry and cosmetic surgery for about four decades. Even the usefulness of this autologous substance in both these fields seems to be well known. Notably, in treating different cosmetic procedures, PRP is already progressively seen because its high constituents of platelet-derived growth factors and cytokines may improve the biochemical mechanisms correlated with tissue repair and regeneration. After activation, platelets within the PRP secrete various growth factors that exhibit the results of hair regeneration. However, PRP may be prepared with different protocols and procedures. Differences could influence the character and possible effectiveness of the end PRP product in the availability of RBCs and WBCs, the introduction of thrombin or calcium chloride to trigger fibrin initiation, and $\mathrm{pH}$-altering substances. $\mathrm{A}$ standard procedure for PRP preparation and application and a framework to assess results have not been developed, though many research works have been published. Thereby, the subsequent research article presents a proposed treatment protocol for AGA by platelets-rich plasma (PRP), which has been confirmed after reviewing different studies performed by researchers that exhibit PRP's efficacy in AGA.

Keywords: Androgenic alopecia, platelet-rich plasma, platelet-derived growth factors, cytokines, cosmetic surgery.

\section{INTRODUCTION}

Hair loss is a common problem that ascends with age in both males \& females. Though several triggering factors causing such behavior in both the genders differ in some parameters, main pin factors are similar. Stress, hormonal imbalances \& hereditary influences are amongst the most ordinary dynamics that provoke hair loss in both genders. Though, the assumed etiology is not a novel concept; but a historical one that has a stronghold onto new levels of grip over the decades in both genders with the aid of other behavioral factors [1].

The medical term for hair loss is devoted to androgenic alopecia. As mentioned, the disease can affect both males \& females with relative severity, sponsored by sexual considerations along with behavioral aspects.

AGA is dispersed hair loss attributable to endocrine and
Published Online: January 8, 2021

ISSN: $2593-8339$

DOI: $10.24018 /$ ejmed.2021.3.1.629

\section{Dr. Sheher Bano*}

Consultant Dermatologist. (e-mail: drsheherbanoipal@gmail.com) Dr. Tariq Mehmood Dar Physician. (e-mail: drtariqdar@gmail.com)

*Corresponding Author hereditary factors. Further, it is defined as the gradual weakening of hair follicles by converting thick, pigmented hairs into barely noticeable hair [2]. Androgenic alopecia is considered to exhibit high levels of 5-alpha reductase in the tissues surrounding the hair follicle. The 5-alpha reductase is responsible for converting testosterone into dihydrotestosterone. Dihydrotestosterone attaches with androgen receptors and causes the induction of genes, which turn stable hair follicles in androgen-based regions of the scalp into thin, weak hair. These regions are middle, frontal, and parietal [3].

\section{ANDROGENIC ALOPECIA IN MALES}

Hair loss in males may be referred to as 'baldness' or androgenic alopecia. The ailment is an ordinary malaise observed in males, as compared to other dermatological 
disorders. As age progresses, many males suffer from alopecia. However, by the age of 80 , almost $80 \%$ of males suffer from alopecia with excess severity levels, resulting in shattering of their confidence levels [4]. Apart from stress level, males tend to catch this dermatological malady by their level of hair baldness. Alopecia in Males is globally considered the most witnessed disorder in dermatology [2].

\section{ANDROGENIC ALOPECIA IN FEMALES}

The disorder is a much-known dermatological error in females, majorly contributed by hormonal imbalances, preand post-menopause issues along with high levels of stress. Androgenetic alopecia is estimated to witness by $10 \%$ of females in their pre-menopause duration, and the remaining percentage receives AGA after they cross the age of 65 [3]. However, prediction of AGA in females is not easy, as, in comparison to males, females go through much complicated hormonal processes, including fluctuating levels of estrogens. Female pattern hair loss is a reserved term for females suffering from such a phase as naming hair loss process in females and males helps understand the treatment procedures. By far, studies have researched the fact that treatment procedures of AGA differ for males \& females and are still debating upon it [5].

\section{HAIR AGING SCIENCE}

Aging \& care of hair is a much-underrated matter that demands attention \& attention measures as individuals show severity in such mediums, and cases of novel hair scalp disorders are reporting as time is passing [6]. In comparison to skincare aging, damaging itself, breaking out, resulting in rashness, swelling, and other dermatological concerns, the care of hair scalp is not loudly treated [7]. Nonetheless, as the cosmetic industry is growing and working in this particular medium, where they launch different hair styling tools and customers, out of curiosity, try them [8]. Reportedly, $65 \%$ of patients who suffer from hair loss blame a specific hair styling tool for damaging their hair. However, fashion dyes and hair treatments do majorly participate in this worth-taking race, individuals get hair loss significant due to them. [9].

Hair-aging cannot be entirely blamed upon such external parameters as biological concerns. Stress levels, and different developmental factors also push this process off a cliff. Though, treatments regarding reversing the process of hair-aging in individuals are ultimately focusing it be a reversible $\&$ in less aged one [10].

\section{HAIR LOSS AND GROWTH ANALOGY}

Hair loss or baldness is a serious concern prevailing worldwide for decades [11]. Though, the severity of this disease has amplified with specific hair treatments \& procedures in the present decade. Hair is the fastest growing tissue in the human body and hair scalp [12]. Hair follicles undergo extremely damaging \& challenging traumas respectively in males \& females' bodies. However, this phenomenon does not stop or abide by here environmental factors. The nature of water used to wash or treat the hair majorly affects the current hair condition, especially the scalp [13]. The hair follicle is made up of key constituent elements that provoke or limit the hair fall, amongst which dermal papilla is the most influential one. Cell activation of hair follicles is controlled by the internal chemical regulators [14]. The weakening of the most dominant component in the upbringing of hair growth initiates hair loss [15].

While hair growth is also contributed by several steps making up a healthy hair follicle [16], unknowingly, hairs play a pretty vital role in building the confidence of an individual, despite cultural \& social values.

The hair growth industry currently holds $2.8 \$$ Billion products, but none can regenerate the already gone hair [17]. Complete reversing of lost hair, with aid from hair loss products, is not proven successful. However, new hair could be generated through such means. AGA is the muchprevailed disease when baldness or hair loss starts in an individual [18]. AGA weakens the hair follicles by shifting their maturity state through a fast-paced terminal, resulting in heavy hair loss. Certain methods have been discovered to reduce baldness that uses dermal papilla (DP) and the influx $\&$ efflux of cells and enhance its size during the hair cycle [19].

\section{PlatelET-RICH PLASMA}

Accordance lacks the correct \& righteous brief of plateletrich plasma [20]. PRP is denoted as a platelet-rich distillate with higher than baseline heights of platelets equaled with whole blood. It provides much vast help in tissue regeneration \& healing [21]. Platelets-rich plasma is considered to be a capacity of autologous plasma, which entails a platelet deliberation overhead basal attentiveness (150 000-350 000/_L) [22].

PRP is made an amalgamation of platelets, cytokines, growth factors, and leukocytes, which results in the birth of certain various nomenclatures [23]. These nomenclatures of constituents' value vary according to the several fractions that are obtained in results of different methods such as Platelet-poor Plasma (PPP), platelet-rich fibrin matrix (PRFM), growth-factors rich plasma (GFRP), leukocyte-rich platelet-rich plasma (LR-PRP), platelet-rich plasma (PRP), and leukocyte-poor platelet-rich plasma (LP-PRP).

\section{PlATELET-RICH PLASMA - IMPLICATION}

There are enormous researches on platelet-rich plasma which explain its efficacy in different field of medicine. Many systematic reviews have been conducted to support the entitled statement, which has been put forward after practical experiments on living organisms. However, activated platelet-rich plasma is considered a potential contender for curing bone tissues [24].

A timeline was also proposed by PRP's implication in several fields, illustrated in figure-1 [25]. PRP has been a supporting agent in different medicinal fields, ranging from mixing in other inhibitors to its solo debut in curing diseases. PRP is proven to be beneficial in such cases. 
Further discussions in the upcoming matter regarding PRP's use for treating alopecia will more upkeep the entitled statement.

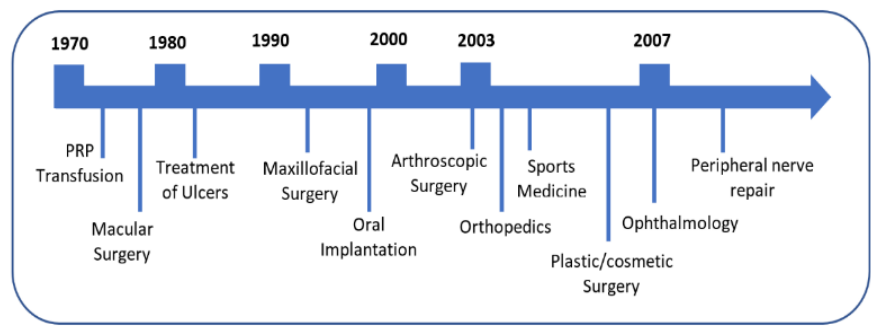

Fig. 1. Chronological sequence of PRP implications in several dimensions of medicine [25].

\section{CONSTITUENTS OF PLATELET-RICH PLASMA}

The illustrated depiction in figure-2 defines the constituent of PRP necessary for a successful outcome in the form of an activated platelet-rich plasma [26].

Motioning proteins of PRP are divided into adhesive proteins \& proteases, growth factors, cytokines \& chemokines. These proteins in amalgamation with tiny molecules \& ions obtained from platelets discharge react with several types of cells. Thereby, constituents of PRP react with cells from the immune response and disparity, angiogenesis \& inflammation. Platelets, in an active state, are more efficiently utilized to eject several growth factors along with cytokines with aid from their alpha granules to cater the angiogenesis \& wound healing process. Growth factors-rich plasma may become dynamic when transmitted onto the scalp through injections that could initiate hair growth.

The growth factors released depict a vital part in the activation of fibroblast, spur of the extracellular matrix, collagen production \& above limits display of endogenous growth factors. Platelet-derived growth factors (PDGF), TGF- $\beta$ - (transforming growth factor beta), along with VEGF- Vascular endothelial growth factor, IGF-1-Insulin like growth factor-1, FBF-fibroblast growth factor \& EGF known as the epidermal growth factor are some of the important constituents of platelet's alpha granule [27].

The exact and standard PRP mechanism to provoke hair growth is yet to discover and define accordingly. Consequently, activated PRP is meant to influence hair cycling by extending the anagen phase's tenure \& delaying apoptosis \& catagen phase [28]. However, enhanced vascularization \& angiogenesis of hair follicles are believed to be technical for inaugurating the anagen phase [29]. Contrariwise, diminished pressure of oxygen \& bloodstream is witnessed in alopecia [4].

Thereby, PRP's activated growth factors in the bulge area of follicles react to cause folliculogenesis \& neovascularization [30].

Hence, growth factors-rich plasma might help to promote vascularization, delay apoptosis \& in so doing extending the anagen phase of hair follicles.

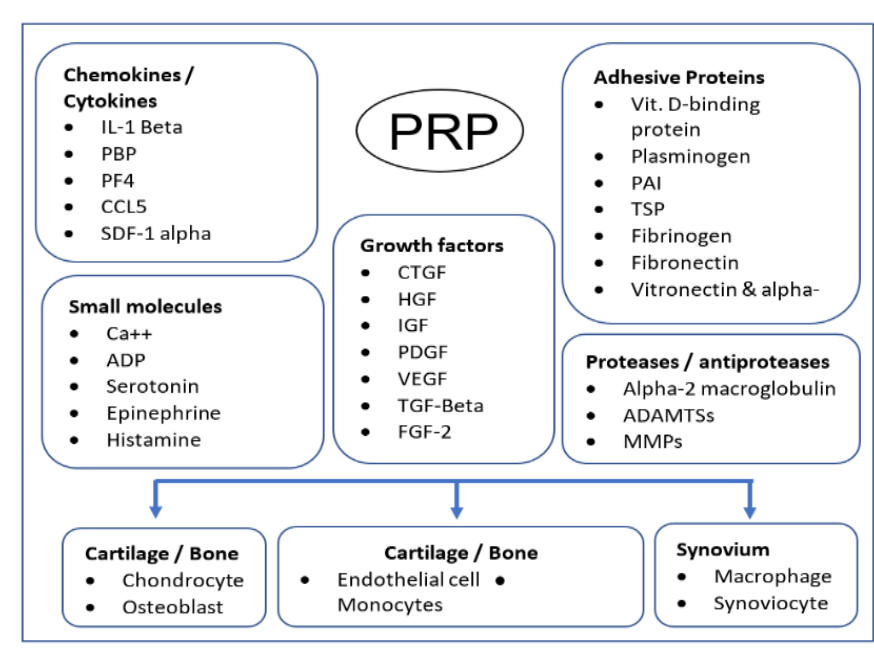

Fig. 2. Constituents of PRP [26].

Abbreviations: IL, Interleukin; PBP, Platelet basic protein; PF 4, Platelet factor 4; CCL5, Chemokine ligand 5; SDF, Stromal cell derived factor; ADP, Adenosine diphosphate; CTGF, Connective tissue growth factor; HGF, Hepatocyte growth factor; IGF, Insulin like growth factor; PDGF, Platelet derived growth factor; VEGF, Vascular endothelial growth factor; TGF, Transforming growth factor; FGF, Fibroblast growth factor; PAI, Plasminogen activator inhibitor; TSP, Thrombospondin; ADAMTSs, a disintegrin and metalloproteinase with thrombospondin motifs; MMPs, Matrix metalloproteinases.

\section{PRP CLASSIFICATION SYSTEM}

Clinical bits of advice have been registered from numerous researchers about the recognized \& functional attributes to stimulate PRP's calibration. These suggested calibrations aid in the relationship \& elucidation of multiple researchers. Presently, PRP entails a variety of characteristics within itself that are not genuinely categorized in its premises, including the presence or nonexistence of WBCs, meditation of platelets, along with several dynamics that correspondingly affect hair development. In the on-going matter, a classification scheme for PRP, named the DEPA, has been suggested [31]. According to the researcher's scheme, the dose, efficiency, purity \& activation of PRP describes the creation's efficiency, activation of the platelets, the dose of injected platelets, and PRP's purity. The DEPA process initiates from the injected platelet dose acquired when multiplication of the present platelet deliberation in PRP by the PRP's attained tome is performed. The dose of platelets being injected is advised to be evaluated before in units of millions or billions. Platelets mended in the PRP (in percentage) from blood testers describe the produced material's efficacy.

Furthermore, the PRP purity indicates the qualified amalgamation of red blood cells (RBCs), platelets, and white blood cells (WBCs). Lastly, the "A" in DEPA confirms the addition of platelet activators, which are exogenous in nature, including calcium chloride, thrombin \& calcium gluconate. According to another classification ritual, the attentiveness or absence of WBCs, platelet dilution \& overall activation eminence of the process is carried out after the unified comparison of it with the baseline blood as a whole [32]. Conversely, another research focusing on the PRP classification module, where the researchers depicted the system with the affirmation of fibrin, its presence and absence [33]. 
There is viable presence of modules into four classified families: L-PRP (Leukocyte \& platelet-rich plasma), L-PRF (Leukocyte \& platelet-rich fibrin), P-PRF (Pure platelet-rich fibrin), and P-PRP (Pure platelet-rich plasma). Another study demands attention [34]. As per the researcher's suggested classification module, the PRP systems present since 2006 comprising single spin postponement \& buffy pelt are vital. Furthermore, a double spin method is also proposed, which claimed a higher platelet calibration than the whole platelet deliberation present in the blood [34].

\section{PRP-MECHANISM OF ACTION}

The exact mechanism regarding PRP's action is still yet to be researched; however, several researchers have conducted studies to discuss the particular matter. Nonetheless, the nature of PPR growth factors \& other constituents needs to be addressed first. Several growth triggers \& cytokines from alpha granules discharged from activated platelets have a role in healing process. Though, numerous growth factors are discharged when platelets in PRP come in an activation state onto the hair scalp, which eventually helps in the evolution of new hair. The released growth factors display a significant part in the activation of collagen blending, fibroblast functioning, imitation of the extracellular matrix, and angiogenesis. Hair evolution using the vivo \& in vitro models for PRP has been researched [28]. Propagation of dermal papilla (DP) has been induced by activated PRP, which happens with the activation of protein kinase $B$, known as an antiapoptotic motioning molecule [27], [28], [35].

However, platelet-derived growth factor (PDGF), vascular endothelial growth features (VEGF), insulin-like growth factor-1 (IGF-1) and transforming growth factorbeta (TGF- $\beta$ ) are discharged by PRP's activated platelets [27]. These discharged growth factors are affirmed to enhance angiogenesis, propagation of cells, which are vital constituents for the evolution of new hair [36], [37]. Minimized free oxygen developed factors and blood flows are conditions in AGA [4]. Stem cells are found to be stimulated by the growth factors derived from activated platelets along in the inflammatory area of hair follicles, and folliculogenesis is observed due to neovascularization [28], [30]. The current thoughts indicate PRP's role in attaining the hair follicles' vascularization and hindering apoptosis, which will eventually elongate the anagen phase of hair growth by striving it for a faster pace in the dermal papilla (DP) cells.

\section{PREPARATION OF PRP}

A uniform yet the primary method of preparing PRP is mentioned in all the PRP protocols. The majority of these processes initiate blood collecting with an appropriate anticoagulant (e.g., sodium citrate, etc.) to stop sudden blood clotting. Plasma, along with platelets, could be extracted after centrifugation of collected blood for a specific time. Centrifugation separates platelets with plasma from the rest of blood cells.
However, an effective and standard procedure to prepare and implicate PRP is still not researched. The basic pattern allows PRP development from the process of cell detachment by making it centrifuged. The centrifuged material then gets injected into the AGA portions of the scalp. Numerous studies have attempted to study how to prepare PRP by using handbook techniques \& viable kits. Growth factors of platelets obtained through the procedures may remain active for several days after injection [38]. Simultaneously, the dispersed variation of protocols describes several amalgamations of PRP, including erythrocytes, leukocytes \& other biological constituents [39], [40].

A study introduced an assessment of preparing PRP where leukocytes were confined to be a vital part of the procedure [41]. Additionally, platelet dilution is also defined as the disbursed consideration to affect PRP efficiency [31].

Conversely, based on studies, the presence of leukocytes is not advised to incur in PRP [42], [43].

Lastly, the detachment of erythrocytes in most PRP preparing techniques is loudly highlighted because evidence exists about them being a factor of discharging oxygen species \& further aid in stress promptness [31].

Apart from all the necessary constituents \& standard procedure to prepare PRP, leukocyte, erythrocyte, platelets' richness, and platelet activation are the definite chief bounds affecting PRP.

\section{DISCUSSION}

To fulfill this article's intended objective, manifold successful clinical research has been incurred in the subsequent review material's encoded discourse. These studies depict the efficacy of PRP through the treating procedure of AGA. A comparison between different kinds of literature is difficult to deduce because of the standardization lacking administration \& dose protocols in the studies, proceeding further with the consistent dimension scales \& method of treatments used.

A cohort study was presented to compare the PRP relevancy for curing AGA [44]. For the purpose of fulfillment, 20 AGA Patients were selected \& treatment was carried out accordingly. At a three-week interval, every patient was treated with three sittings of PRP \& one at an interval of six months. Hair density was increased as an outcome of the treatment \& was amplified in the third month to the most. Whereas hair density was lessened at the sixth \& twelfth month proceeding the treatment but managed to be above baseline. Thereby, an additional treatment was recommended during the sixth month to sustain the achieved outcomes. The study mentioned that AGA patients with less severe AGA recovered quickly with PRP than those with relatively severe AGA.

Likewise, another study was performed to discover the ultimate benefits of inoculating platelet growth factors into patients' scalp [45]. Leukocyte-PRP (L-PRP) was extracted for injection upon 64 AGA patients. Moreover, two interventions were also included carrying plasmatic proteins on patients' hair scalps by a gap of 3 months. The assessment was performed through clinical photographs. 
Another clinical study was conducted to measure the worth of PRP in considering AGA [46]. The researchers selected ten AGA patients and administered the PRP procedure for three months. For three months, every twothree weeks, the sessions of PRP treatments were managed for further evaluation. After that, the Pull test displayed particular results \& hair thickness, hair count \& degree of alopecia was testified. The study concluded the trial by deducing PRP to be the most cost-effective \& imperative treatment for AGA.

Another controlled study was conducted on a population of 25 patients suffering from androgenic alopecia (AGA) [27]. The patients included in the research were enclosed upon their non-relevancy with any medicinal drugs \& belonged from stage I to III of Ludwig scale along with Stage II to Stage V of Hamilton-Norwood scale. All the patients included in the research attended three PRP sittings and were finally evaluated utilizing photography of phototrichogram.

In comparison to the criterion, particular progress was observed statistically in the mean telogen hairs, anagen hairs, terminal hair density, and hair density (itself) in areas treated with PRP on the sixth month. As stated, the other half of the study included patients who received treatment in a placebo manner. Thereby, compared to the control (placebo) group, the intervention group (who received the actual treatment) showed a particular statistically enlarging hair density value continuously. The researchers suggested three preliminary treatments for AGA patients proceeding with a month gap in between. As per the researchers, the treatment could be concluded after a six-month gap of initial three treatments.

Similarly, a study on males was carried out, included ten male AGA patients [35]. It was ensured that the patients were not taking any other medicinal drugs for the earlier 12 months. Likewise, to the two studies discussed above $\&$ an inclusion criterion of the following article's literature, the research under discussion was also a blinded, randomized one, along with the half-head assembly considering with placebo. Researchers used autologous activated platelet-rich plasma in their study. Evaluations were performed three months later to the treatment sessions, and a mean enlargement of 27.7 hairs/cm2 was observed in the PRP treated areas. The entitled observation was conceded out after comparison with control areas, where a decrease of three hairs/cm2 was detected.

In a further study, researchers steered a surveyor blinded, randomized, and placebo organized research setting [43]. The following study's inclusion area was roamed around to only have males on hair loss stages ranging from II to IV as per the classification of Norwood-Hamilton. Thereby, 20 male AGA patients were involved in carrying out different outcomes.

Evaluations were conducted after the interludes of a month, subsequently to at least three sittings of PRP. Outcomes were deducted in a comparison of the placebo group with the intervention group \& patients of the latter group showed an improvement in total hair density and amplified amount of hair count, which was assessed by a computerized Trichogram.
Before announcing the final verdict, the researchers carried out additional immunohistochemistry \& microscopic estimation after two weeks of accomplishing PRP treatment. Researchers witnessed an increment in mini blood vessels around hair follicles, epidermal basal keratinocytes \& lump cells of the hair follicle in PRP-treated hair skin through the additional evaluation. In conclusion, researchers recommended PRP for enhancing follicular angiogenesis \& keratinocyte, to control an individual's hair loss.

In another clinical study, the researchers performed the PRP treatment on patients suffering from alopecia, and during the whole discourse of treatment, patients received five injections [47]. The injections contain 2, 3, and $4 \mathrm{ml}$ of PRP material. PRP contained in the injected injections was prepared from a single spin process. The assessment was conducted three months preceding the accomplishments of treatments. The researchers concluded that a diminishing amount of anagen to telogen ratio along with decreased anagen was observed. However, an increment was observed in telogen, which concludes further the PRP's inefficacy for treating AGA because not any change in density of hair or even in the count was observed.

In another study, twenty-six females AGA patients were selected with a Ludwig II score of AGA [48]. The study was double-blinded \& conducted in a placebo-controlled environment. The study concluded that hair count did not enhance on comparing both the placebo \& PRP treated patients' group. Conversely, patients' opinions such as ease in styling hair (after the treatment) were observed upon patients' surveys.

Another study established that activated PRP has antiapoptotic properties [49]. It enhances hair follicles' endurance and could excite hairs by extending the hair cycle's anagen phase. They added that activated PRP exhibits the efficacy that stimulates DP cells' proliferation.

Theoretically \& practically, seven out of nine reviewed studies mentioned \& proved PRP's efficacy in treating AGA. Though there are not any long-term results are reported, which further opens a window for future studies. However, the failed impact of PRP for AGA [47][48] attracts necessary regard to the fact that assessments must be mainly made to check in adherence to PRP to conclude it as an effective means for treating AGA. The reviewed literature can also be utilized for opening new paths to measure the efficacy of PRP.

\section{MATERIAL \& METHODS}

\section{A. Patients}

A total of three male patients with age range 38-45 years had been selected with AGA. Patients on any topical or systemic treatments were excluded. Immunosuppressed patients were also not qualified for our study. The identification of AGA was clinically recognized \& established. All participants of the study signed a written consent before the study start.

\section{B. Treatment Protocol}

Activated autologous platelet-rich plasma was made from $18 \mathrm{~mL}$ of each participant's blood sample as per the method of the Prizmah system (AK Pharma Pvt. Ltd.). In short, to 
have PRP, blood was drawn from each participant into two blood-collecting sterilized tubes through a butterfly needle. Blood collecting tubes were vacuumed only for $9 \mathrm{~mL}$ blood having sodium citrate as an anticoagulant and a gel separator. After collecting the blood, both tubes were placed in a centrifuge machine for 7 minutes at the speed of 3000 rpm. It was a single spin procedure as defined by the Prizmah system. After 7 minutes spin, autologous PRP was obtained with no RBCs and only almost $10 \%$ of WBCs.

Nearly $10 \mathrm{~mL}$ of pure autologous PRP was extracted with this system $(5 \mathrm{~mL}$ of autologous PRP from each $9 \mathrm{~mL}$ of blood). The last step to have platelets growth factor out of alpha granules. Growth factors start to excrete from alpha granules when platelet activation starts. For this, the Prizmah system contained $10 \mathrm{~mL}$ sterilized tubes with 0.03 $\mathrm{mL}$ calcium chloride. Prepared pure autologous PRP has then transferred into calcium chloride-containing tubes through a closed system transfer device. Then activated PRP, may be stated now as "growth factors-rich plasma", transferred into $1 \mathrm{~mL}$ syringes through a closed system transfer device. By this procedure, we have autologous growth factors-rich plasma with $\mathrm{pH}$ within the blood range (7.4 approx.).

The injections were spread out in the scalp's thinning area, which was basically along the bald hairline. The patients' selected area of scalp was cleansed with $70 \%$ alcohol (no local anesthesia). The growth factors-rich plasma was introduced on the scalp's pre-described area with $0.1 \mathrm{~mL} / \mathrm{cm}^{2}$ quantity.

Three treatment sessions were applied at first, second, and third months respectively, and then analyzed. Generally, participants with AGA have promising results from growth factors-rich plasma applications noticed after three sessions (one session every month) regarding hair growth, hairs' improved density, and enhanced quality of life (Fig.3, 4 \& 5).
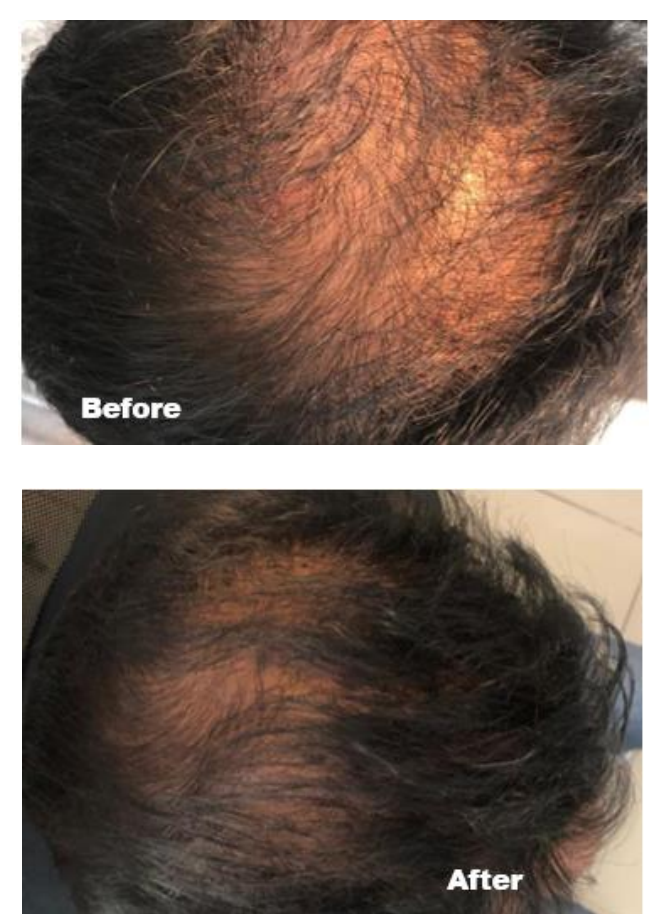

Fig. 3. A man with androgenic alopecia (42 years old), before and after 3 treatment sessions of Prizmah-PRP (Growth factors-rich plasma) (One session every month).
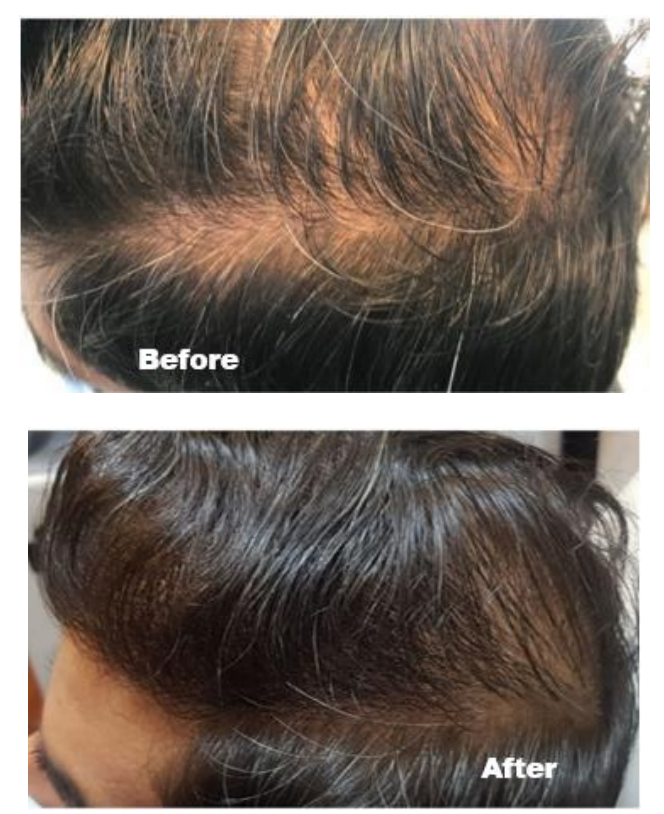

Fig. 4. A man with androgenic alopecia (38 years old), before and after 3 treatment sessions of Prizmah-PRP (Growth factors-rich plasma) (One session every month).
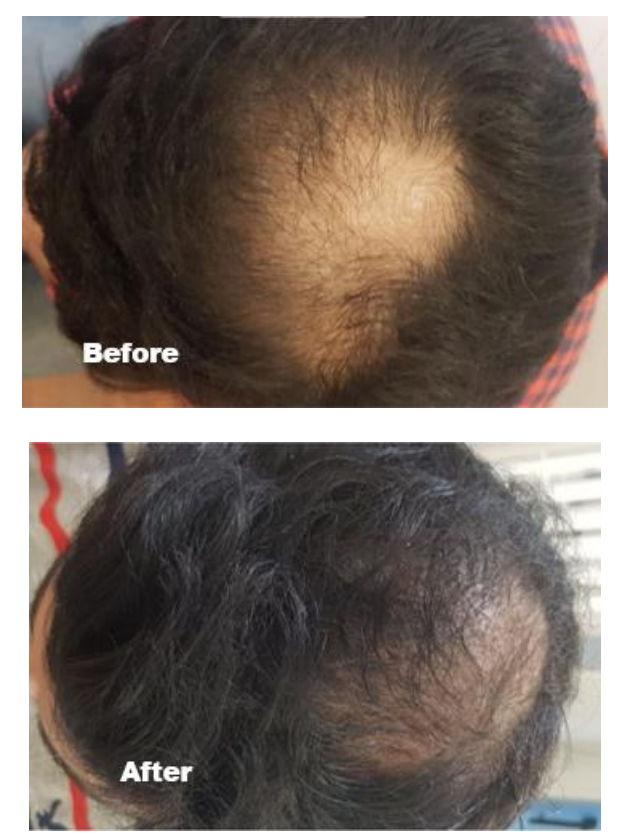

Fig. 5. A man with androgenic alopecia (45 years old), before and after 3 treatment sessions of Prizmah-PRP (Growth factors-rich plasma) (One session every month).

\section{CONCLUSION}

Platelet-rich plasma is a pretty diversified agent when it comes to healthcare. Enormous fields are taking advantage of the activism approach generated through the platelet-rich plasma, including orthopedics, dermatology, and tissue regeneration, as proven through reviewed literature. The subsequent review article demonstrates the maximum relevancy of PRP as a means to treat androgenic alopecia (AGA). Thereby, opting for activated PRP (growth factorsrich plasma) treatment to entertain AGA is beneficial, as mentioned in the clinical studies reviewed. Moreover, side effects \& safety concerns are observed to be minimal. However, activated PRP itself has proven to be an efficient and effective procedure in terms of dosage and interval handling. This paper also exhibits the successful application and outcome of growth factor-rich plasma in selected AGA 
patients. The treatment protocol is proposed as treatment sessions at the first, second, third, sixth, and twelfth month through this study. Afterward, one treatment session every year as a maintenance treatment is being proposed.

For future studies, absolute affirmations are required to clear out the doubts. These affirmations range from the concentration of platelets in PRP, frequency of injections need to treat an AGA patient, any supportive therapeutic options, and extensive tenure follow-ups, which will help determine the longevity of growth factors-rich plasma or activated PRP treatments.

\section{DISCLOSURE}

The authors declare the following competing financial interest(s):

Dr. Sheher Bano declares no conflicts of interest in developing and publishing this study.

Dr. Tariq Mehmood Dar is the Director in AK Pharma Pvt. Ltd.

\section{REFERENCES}

[1] Bertoli, Mia J., Roxanna Sadoughifar, Robert A. Schwartz, Torello M. Lotti, and Camila K. Janniger. "Female pattern hair loss: A comprehensive review." Dermatologic Therapy July (2020).

[2] York, Katherine, Nekma Meah, Bevin Bhoyrul, and Rodney Sinclair. "A review of the treatment of male pattern hair loss." Expert opinion on pharmacotherapy 21, no. 5 (2020): 603-612.

[3] Rogers NE, Avram MR. Medical treatments for male and female pattern hair loss. J Am Acad Dermatol 2008; 59(4):547-66.

[4] Goldman BE, Fisher DM, Ringler SL. Transcutaneous PO2 of the scalp in male pattern Baldness: A new piece to the puzzle. Plast Reconstr Surg 1996; 97(6):1109-16.

[5] Urysiak-Czubatka, Izabela, and Grazyna Broniarczyk-Dyla. "Examination of hair growth parameters in androgenetic alopecia in women using TrichoScan." Postepy Dermatologii Alergologii 27, no. 4 (2010): 246 .

[6] Breitkopf, Trisia, Gigi Leung, Mei Yu, Eddy Wang, and Kevin J. McElwee. "The basic science of hair biology: what are the causal mechanisms for the disordered hair follicle?" Dermatologic clinics 31, no. 1 (2013): 1-19.

[7] Vogt, Annika, and Ulrike Blume-Peytavi. "Selective hair therapy: bringing science to the fiction." Experimental dermatology 23, no. 2 (2014): 83-86

[8] Gerber, Markus, Serge Brand, Magnus Lindwall, Catherine Elliot, Nadeem Kalak, Christian Herrmann, Uwe Pühse, and Ingibjörg H Jonsdottir. "Concerns regarding hair cortisol as a biomarker of chronic stress in exercise and sport science." Journal of sports science \& medicine 11, no. 4 (2012): 571.

[9] Garza, Luis A., Yaping Liu, Zaixin Yang, Brinda Alagesan, John A. Lawson, Scott M. Norberg, Dorothy E. Loy et al. "Prostaglandin D2 inhibits hair growth and is elevated in bald scalp of men with androgenetic alopecia." Science translational medicine 4, no. 126 (2012).

[10] Guerra-Tapia, A., and E. Gonzalez-Guerra. "Hair cosmetics: dyes." Actas Dermo-Sifiliográficas (English Edition) 105, no. 9 (2014) 833-839.

[11] Avci, Pinar, Gaurav K. Gupta, Jason Clark, Norbert Wikonkal, and Michael R. Hamblin. "Low-level laser (light) therapy (LLLT) for treatment of hair loss." Lasers in surgery and medicine 46 , no. 2 (2014): 144-151.

[12] Fox, James G., Stephen Barthold, Muriel Davisson, Christian E Newcomer, Fred W. Quimby, and Abigail Smith. The mouse in biomedical research: normative biology, husbandry, and models. Vol. 3. Elsevier, 2006.

[13] Paus, Ralf, and Kerstin Foitzik. "In search of the "hair cycle clock": a guided tour." Differentiation 72, no. 9-10 (2004): 489-511.

[14] Tiede, Stephan, Jennifer E. Kloepper, Enikö Bodò, Sanjay Tiwari, Charli Kruse, and Ralf Paus. "Hair follicle stem cells: walking the maze." European journal of cell biology 86, no. 7 (2007): 355-376.
[15] Nabahin, Amal, Alaa Abou Eloun, and Samy S. Abu-Naser. "Expert System for Hair Loss Diagnosis and Treatment." International Journal of Engineering and Information Systems, Vol. 1 Issue 4, June- 2017, Pages: 160-16

[16] Trüeb, Ralph M., Jim P. Henry, Mike G. Davis, and Jim R. Schwartz. "Scalp condition impacts hair growth and retention via oxidative stress." International journal of trichology 10, no. 6 (2018): 262.

[17] Choi, Bu Young. "Hair-growth potential of ginseng and its major metabolites: A review on its molecular mechanisms." International journal of molecular sciences 19, no. 9 (2018): 2703.

[18] Wolff, Hans, Tobias W. Fischer, and Ulrike Blume-Peytavi. "The diagnosis and treatment of hair and scalp diseases." Deutsches Ärzteblatt International 113, no. 21 (2016): 377.

[19] Pantelireis, Nikolaos, and Claire A. Higgins. "A bald statementCurrent approaches to manipulate miniaturisation focus only on promoting hair growth." Experimental dermatology 27, no. 9 (2018): 959-965.

[20] Baksh, N, Hannon, CP, Murawski, CD, Smyth, NA, Kennedy, JG. Platelet-rich plasma in tendon models: a systematic review of basic science literature. Arthroscopy. 2013; 29:596-607.

[21] Fitzpatrick, Jane, Max K. Bulsara, Paul Robert McCrory, Martin D. Richardson, and Ming Hao Zheng. "Analysis of platelet-rich plasma extraction: variations in platelet and blood components between 4 common commercial kits." Orthopaedic Journal of Sports Medicine 5, no. 1 (2017).

[22] Wroblewski AP, Melia HJ, Wright VJ. Application of platelet rich plasma to enhance tissue repair. Oper Tech Orthop.2010; 20:98-105.

[23] Masuki, Hideo, Toshimitsu Okudera, Taisuke Watanebe, Masashi Suzuki, Kazuhiko Nishiyama, Hajime Okudera, Koh Nakata, Kohya Uematsu, Chen-Yao Su, and Tomoyuki Kawase. "Growth factor and pro-inflammatory cytokine contents in platelet-rich plasma (PRP), plasma rich in growth factors (PRGF), advanced platelet-rich fibrin (A-PRF), and concentrated growth factors (CGF)." International journal of implant dentistry 2, no. 1 (2016): 1-6.

[24] Kazem-Arki, Mandana, Mahboubeh Kabiri, Iman Rad, Nasim Hayati Roodbari, Hoorieh Hosseinpoor, Samaneh Mirzaei, Kazem Parivar, and Hana Hanaee-Ahvaz. "Enhancement of osteogenic differentiation of adipose-derived stem cells by PRP modified nanofibrous scaffold." Cytotechnology 70, no. 6 (2018): 1487-1498.

[25] E. Conde Montero, a, M.E. Fernández Santos, b R. Suárez Fernández. "Platelet-Rich Plasma: Applications in Dermatology" Actas Dermosifiliogr. 2015; 1062(2):104-111.

[26] Isabel Andia, Nicola Maffulli, Platelet-rich plasma for managing pain and inflammation in osteoarthritis, Nature Reviews Rheumatology $\cdot$ Vol.9, Dec-2013.

[27] Rubina Alves, and Ramon Grimalt. "Randomized placebocontrolled, double-blind, half-head study to assess the efficacy of platelet-rich plasma on the treatment of androgenetic alopecia." Dermatologic Surgery 42, no. 4 (2016): 491-497.

[28] Li, Weiwei, Mitsuhiro Enomoto, Madoka Ukegawa, Takashi Hirai, Shinichi Sotome, Yoshiaki Wakabayashi, Kenichi Shinomiya, and Atsushi Okawa. "Subcutaneous injections of platelet-rich plasma into skin flaps modulate proangiogenic gene expression and improve survival rates." Plastic and reconstructive surgery 129, no. 4 (2012): 858-866.

[29] Mecklenburg, Lars, Desmond J. Tobin, Sven Müller-Röver, Bori Handjiski, Gunnar Wendt, Eva MJ Peters, Susanne Pohl, Ingrid Moll, and Ralf Paus. "Active hair growth (anagen) is associated with angiogenesis." Journal of Investigative Dermatology 114, no. 5 (2000): 909-916.

[30] Takikawa, Megumi, Shinichiro Nakamura, Shingo Nakamura, Masayuki Ishirara, Satoko Kishimoto, Kaoru Sasaki, Satoshi Yanagibayashi, Ryuichi Azuma, Naoto Yamamoto, and Tomoharu Kiyosawa. "Enhanced effect of platelet-rich plasma containing a new carrier on hair growth." Dermatologic Surgery 37, no. 12 (2011) 1721-1729.

[31] Magalon, J., A. L. Chateau, B. Bertrand, M. L. Louis, A. Silvestre, L. Giraudo, J. Veran, and F. Sabatier. "DEPA classification: a proposal for standardising PRP use and a retrospective application of available devices." BMJ open sport \& exercise medicine 2, no. 1 (2016).

[32] Mishra, Allan, Kimberly Harmon, James Woodall, and Amy Vieira. "Sports medicine applications of platelet rich plasma." Current pharmaceutical biotechnology 13, no. 7 (2012): 1185-1195.

[33] M Dohan Ehrenfest, David, Tomasz Bielecki, Ryo Jimbo, Giovanni Barbe, Marco Del Corso, Francesco Inchingolo, and Gilberto Sammartino. "Do the fibrin architecture and leukocyte content influence the growth factor release of platelet concentrates? An evidence-based answer comparing a pure platelet-rich plasma (P- 
PRP) gel and a leukocyte-and platelet-rich fibrin (L-PRF)." Current pharmaceutical biotechnology 13, no. 7 (2012): 1145-1152.

[34] Mautner, Kenneth, Gerard A. Malanga, Jay Smith, Brian Shiple, Victor Ibrahim, Steven Sampson, and Jay E. Bowen. "A call for a standard classification system for future biologic research: the rationale for new PRP nomenclature." PM\&R 7, no. 4 (2015): S53S59.

[35] Cervelli, V., S. Garcovich, A. Bielli, G. Cervelli, B. C. Curcio, M. G. Scioli, A. Orlandi, and P. Gentile. "The effect of autologous activated platelet rich plasma (AA-PRP) injection on pattern hair loss: clinical and histomorphometric evaluation." BioMed research international 2014.

[36] Kim, Dae Hun, Young Jin Je, Chang Deok Kim, Young Ho Lee, Young Joon Seo, Jeung Hoon Lee, and Young Lee. "Can plateletrich plasma be used for skin rejuvenation? Evaluation of effects of platelet-rich plasma on human dermal fibroblast." Annals of dermatology 23, no. 4 (2011): 424-431.

[37] Uebel, Carlos Oscar, Jefferson Braga da Silva, Denise Cantarelli, and Pedro Martins. "The role of platelet plasma growth factors in male pattern baldness surgery." Plastic and reconstructive surgery 118, no. 6 (2006): 1458-1466.

[38] Senzel, Lisa, Dmitri V. Gnatenko, and Wadie F. Bahou. "The platelet proteome." Current opinion in hematology 16, no. 5 (2009): 329.

[39] Ehrenfest, David M. Dohan, Lars Rasmusson, and Tomas Albrektsson. "Classification of platelet concentrates: from pure platelet-rich plasma (P-PRP) to leucocyte-and platelet-rich fibrin (LPRF)." Trends in biotechnology 27, no. 3 (2009): 158-167.

[40] Mazzocca, Augustus D., Mary Beth R. McCarthy, David M. Chowaniec, Mark P. Cote, Anthony A. Romeo, James P. Bradley, Robert A. Arciero, and Knut Beitzel. "Platelet-rich plasma differs according to preparation method and human variability." JBJS 94 , no. 4 (2012): 308-316.

[41] Giusti, Ilaria, Marianna Di Francesco, Sandra D'Ascenzo, Paola Palumbo, Anna Rughetti, Luigi Dell'Orso, Paola Annunziata Varasano, Gabriella Lucidi Pressanti, and Vincenza Dolo. "Leukocyte depletion does not affect the in vitro healing ability of platelet rich plasma." Experimental and therapeutic medicine 15, no. 4 (2018): 4029-4038.

[42] Weibrich, G., T. Hansen, W. Kleis, R. Buch, and W. E. Hitzler. "Effect of platelet concentration in platelet-rich plasma on periimplant bone regeneration." Bone 34, no. 4 (2004): 665-671.

[43] Gentile, Pietro, Simone Garcovich, Alessandra Bielli, Maria Giovanna Scioli, Augusto Orlandi, and Valerio Cervelli. "The effect of platelet-rich plasma in hair regrowth: a randomized placebo-controlled trial." Stem cells translational medicine 4, no. 11 (2015): 1317-1323.

[44] Gkini, Maria-Angeliki, Alexandros-Efstratios Kouskoukis, Gregory Tripsianis, Dimitris Rigopoulos, and Konstantinos Kouskoukis "Study of platelet-rich plasma injections in the treatment of androgenetic alopecia through a one-year period." Journal of cutaneous and aesthetic surgery 7, no. 4 (2014): 213 .

[45] Schiavone, Giovanni, Desanka Raskovic, Joseph Greco, and Damiano Abeni. "Platelet-rich plasma for androgenetic alopecia: a pilot study." Dermatologic Surgery 40, no. 9 (2014): 1010-1019.

[46] Singhal, Parul, Sachin Agarwal, Paramjeet Singh Dhot, and Satish K. Sayal. "Efficacy of platelet-rich plasma in treatment of androgenic alopecia." Asian journal of transfusion science 9, no. 2 (2015): 159.

[47] Ayatollahi, Azin, Hamed Hosseini, Majid Shahdi, Saman Ahmad Nasrollahi, Mansour Nassiri Kashani, Somaye Yadangi, Farid H. Firooz, and Alireza Firooz. "Platelet-rich plasma by single spin process in male pattern androgenetic alopecia: Is it an effective treatment?" Indian Dermatology Online Journal 8, no. 6 (2017): 460.

[48] Puig CJ, Reese R, Peters M. Double-blind, placebo-controlled pilot study on the use of platelet-rich plasma in women with female androgenetic alopecia. Dermatol Surg 2016;42(11):1243-7.

[49] Zheng Jun Li, Hye-in Choe, Dae-Kyoung Choi, Kyung-Cheol Sohn, Myung Im, Young-Joon, Young-Ho Lee, Jeung-Hoon Lee, Young Lee, "Autologous Platelet-Rich Plasma: A Potential Therapeutic Tool for Promoting Hair Growth" Dermatol Surg 2012;1-7.

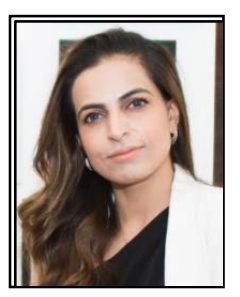

Dr. Sheher Bano is a consultant Dermatologist at IPAL Clinic, Islamabad, Pakistan. She is the pioneer in introducing aesthetic line of treatment at a leading boutique hospital of nation's capital. She completed her MBBS from the Nishter Medical College, Multan, Pakistan, underwent her postgraduate MCPS training in pursuance of her passion of dermatology. She never stopped there and undertook MD Dermatology training at the PIMS, Islamabad in
2015. Her cosmetic training is from Cohere, UK, where she attended multiple courses to enhance her aesthetic skills. Fueled by her passion for skincare and desire for the entrepreneurship, she decided to set up IPAL Clinic, Islamabad, Pakistan, as innovative, relaxed, quality driven and confidential practice fostering all sorts of skincare needs.

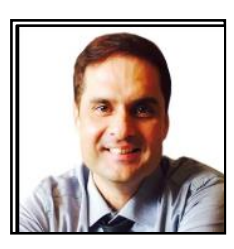

Tariq Mehmood Dar is a medical doctor. He earned his MBBS degree from Dow Medical College, Karachi University, Pakistan. He is a Physician and did his postgraduation in Diabetology. Dr. Tariq is a Clinical Research Certified Professional. Currently, he holds a position of Director Marketing \& Sales in a multinational pharmaceutical company based at UAE. 